\title{
Psychoanalyse, flüchtige Moderne und die Aufgabe des Selbst
}

\author{
Barbara Zielke (Nürnberg)
}

Zusammenfassung: Der Beitrag geht der These nach, dass die veränderten Lebensbedingungen in der Spätmoderne bestimmte bislang bewährte Prädikate des personalen Selbst, wie Kohärenz oder Kontinuität, fraglich werden lassen. Gestützt auf die Kritik der Subjektphilosophie und die Kritik des Individuozentrismus der «westlichen» Psychologie des Selbst werden zunächst jene Positionen beleuchtet, die eine Auflösung des einheitlichen Selbst postulieren. Es wird argumentiert, dass diese Positionen in ihren Interpretationen der spätmodernen Lebensverhältnisse unnötig weit gehen. In der Folge werden daher Vorschläge der intersubjektiven Psychoanalyse vergleichend daraufhin gelesen, inwiefern sie das geeignete begriffliche Instrumentarium aufweisen, um das Selbst im Sinne einer reflexiven Moderne als sozial konstituiert, differenzoffen und intern vielfältig zu begreifen, ohne den Standard des selbstbestimmten (kritischen) Subjekts gänzlich aufzugeben. Zuletzt wird angedeutet, wo die spezifischen Stärken psychoanalytischer Konzeptualisierungen des aus Intersubjektivität emergierenden, «transitorischen» Selbst liegen (könnten): in der Funktion als "Biografiegenerator», in der entwicklungspsychologischen Perspektive, im Pendeln zwischen Theorie und Behandlungssituation sowie in der Bereitschaft zur Relativierung des psychoanalytischen Wissens.

Schlüsselwörter: reflexive Moderne, Globalisierung, Intersubjektivitätstheorie, relationale Pychoanalyse, transitorische Identität 
Jeder Einzelne hat so Anteil an vielen Massenseelen, an der seiner Rasse, des Standes, der Glaubensgemeinschaft, der Staatlichkeit usw., und kann sich darüber hinaus zu einem Stückchen Selbstständigkeit und Originalität erheben. (Sigmund Freud, 1921)

\section{Einleitung}

Das Selbstverhältnis der Individuen hat sich verändert - hierin scheinen sozialwissenschaftliche und psychoanalytische Gesellschaftsdiagnosen einig. Das Heute ist bestimmt von der Allgegenwart kultureller Differenz, von globaler Verflechtung, von verselbstständigten Techniken insbesondere der Kommunikation und einer von allgegenwärtigen Medien gesetzten «Hyperrealität» (Baudrilliard, 1976). Aufgrund dieser und weiterer Charakteristika spätmoderner, globalisierter Gesellschaften stellt sich für die Subjekte die Identitätsfrage neu: Wer bin ich in einer Welt, die es an eindeutigen Orientierungen fehlen lässt, die mich zwingt, permanent zwischen vielfältigen und unterschiedlichen Kontexten zu wechseln, mich mit radikal differenten Lebensformen konfrontiert und mit den vielfältigen irrationalen Folgen rationaler Strategien (Giddens, 1990)? In skeptischem Tonfall schreibt bekanntlich Zygmunt Bauman Mitgliedern postmodernen, globalisierten Gesellschaften zu, sie seien immer «on the move» und daher auch (fast) immer «strangers» (1996, S. 28). Die dort vorgängigen Lebensformen des immer währenden Touristen und andere "postmoderne Lebensstrategien» haben für den Soziologen vorrangig eine Auswirkung: Sie führen dazu, dass soziale Beziehungen «fragmentierter» werden, was wiederum der Hauptgrund dafür ist, dass sich das Selbst heute ganz neuen Anforderungen zu stellen hat: Das postmoderne Problem der Identität, so Bauman, besteht hauptsächlich darin, «die Festlegung zu vermeiden und sich Optionen offen zu halten» (1996, S. 133). Aus denselben Gründen stellt Richard Sennetts (1998) wehmütige Klage den allgemeinen «Verfall des Charakters» fest. Für ihn ist die systemisch bedingte Auflösung der auf Langfristigkeit angelegten personalen Identität und Autonomie eine direkte Folge des Spätkapitalismus.

Fragmentierung, Auflösung, Identitätsverfall: Dies sind sehr skeptische Beobachtungen, auf die die sozialwissenschaftliche Forschung zur personalen Identität ebenso zu reagieren versucht wie die psychoanalytische Begriffsbildung zu Selbst, Selbstgefühl und Identitätsentwicklung. Auch ich möchte im Folgenden 
der Frage nachgehen, wie berechtigt diese Skepsis ist. Können wir tatsächlich nicht mehr von bewährten Identitätsprädikaten wie Kohärenz, Kontinuität oder gar Autonomie sprechen? Können traditionelle psychoanalytische Auffassungen eines einheitlichen Ichs noch als positive Ziele - etwa von Psychotherapien - in Anschlag gebracht werden oder sind sie, wie manche Kritiker postulieren, unzeitgemäss? Wenn wir etwa mit Axel Honneth davon ausgehen, dass die Tendenz der Subjekte wächst, mehr und unterschiedliche «Identitätsmöglichkeiten» zuzulassen (2000, S. 1089): Was ist unter solchen Identitätsmöglichkeiten zu verstehen, und (inwiefern) machen sie als interne Pluralisierung das Selbst als kohärente Einheit obsolet?

Man könnte diese Frage aus der Sicht der Psychoanalyse schnell dahingehend beantworten, dass es ihr nicht darum gehen kann, ein Subjekt bzw. das Selbst zu verabschieden, welches Gegenstand ihrer Metapsychologie und ihrer Praxis ist. Aber auch die Psychoanalyse muss und will sich einstellen auf kulturelle und gesellschaftliche Veränderungen, sie will diese aus ihrer Sicht besser verstehen und zugleich durch ihre Sicht allgemein zu einem besseren, umfassenderen Verständnis beitragen. Sie hat immer mitgehalten bei den sozialwissenschaftlichen Versuchen, einen zeitgemässen «Sozialtypus» zu bestimmen (z. B. Lasch, 1979; Ehrenberg, 1998; Dornes, 2010), davon zeugen aktuell vielfältige Tagungen und Publikationen zur globalisierten, medialisierten, multikulturellen, beschleunigten gesellschaftlichen Wirklichkeit - unter ihnen auch die aktuelle Ausgabe des Journals für Psychoanalyse.

Daher halte ich es für wichtig, zunächst einmal begründeten Annahmen nachzugehen, auf die sich die zitierten Klagen stützen. Zwei grundlegende Argumentationslinien, die den Zusammenhang aufzeigen könnten zwischen einigen sogenannten Folgen der Globalisierung und der personalen Identität finden sich in der Kritik der Subjektphilosophie und in neueren als postkolonial oder transkulturell bezeichneten Forschungen und Diskursen.

\section{Philosophische Subjektkritik und die Hybridisierung des Selbst}

Besonders Michel Foucaults kritische Analysen haben sichtbar gemacht, dass viele unserer bewährten Identitätsprädikate nicht empirisch überholt, sondern normativ problematisch sind. Sie können - für Foucault: sie müssen - verstanden werden als Zurichtung für eine bestimmte Form der «Sorge um sich» (Foucault, 1989). Wir mögen zwar glauben, durch Selbstaufklärung und lückenlose Selbst-Reflexion auf dem Weg zu unserem eigentlichen Selbst und von äusseren Zwängen «frei» zu sein. Tatsächlich aber werden die uns im Zeichen von Freiheit und Selbstbestimmung zugeschriebenen Errungenschaften durch die Etablierung 
entsprechender Institutionen schnell zu Notwendigkeiten, die uns eine bestimmte Variante derVerantwortung für das eigen Leben aufzwingen und uns zu Subjekten moderner Macht- und Kontrolldispositive machen. Selbstbehauptung wird entlarvt als Selbstunterdrückung. Das aufgeklärte, vernunftgeleitete Subjekt wird in seiner absoluten Selbstsetzung zum fruchtbaren Boden für unterschiedliche Gewaltverhältnisse. Vermeintliche Fortschritte in Naturwissenschaft und Technik können umschlagen in unkontrollierbare Bedrohung, die aber nicht mehr als objektive Gefahr von aussen auftritt, sondern als im Zeichen von Technik und Vernunft selbstgemachtes «Risiko» (Beck, 1986). Dies sind nur einige Argumente, welche die Kritiker der Moderne anführen, um den Tod des modernen Subjekts zu beschwören: Vernunftgeleitete, im modernen Sinne «autonome» Individuen mögen zwar nach wie vor zum normativen Standard erhoben werden, sie können sich aber nicht mehr bewähren in einer Welt, deren Sinn dem rationalen Denken, Bewerten und Handeln Einzelner nicht mehr zurechenbar ist.

Hier lässt sich einwenden, diese Subjektkritik bzw. die von ihr abgeleiteten Vorschläge für eine Umorientierung der wissenschaftlichen Betrachtung des Selbst richte sich gegen ein cartesianisches Subjekt der Aufklärung, von dem die modernen Wissenschaften vom Menschen, die moderne Psychologie zumal, seit Langem nicht mehr ausgingen. Für diese wissenschaftliche und angewandte Psychologie hat allerdings Niklas Rose (1989) in prägnanter Weise verdeutlicht, wie der «psy-complex», die institutionelle Umsetzung dieser Sorge um sich, von Aufforderungen zu Wellness, Psychohygiene und Beratung bis zum boomenden Psychotherapiemarkt, nicht zuletzt dazu dient, Personen zur Buchführung über sich selbst zu verpflichten und sie damit zu «regierbaren» Subjekten zu machen. Ähnlich argumentieren heute jene psychologischen Entwürfe, die etwa seit den 90er Jahren unter dem Oberbegriff «sozialkonstruktionistisch» firmieren, und sie haben es nicht versäumt, positive psychologisch informierte Vorschläge zu erarbeiten, die sich von jeder Variante des Subjekts verabschieden. Ich komme darauf zurück.

Die Pointe der philosophischen und sozialwissenschaftlichen Subjektkritik steht auch Pate für die Argumentation postkolonialistischer Diskurse (Said, 1999), die den Hinweis auf Identitätsprobleme des modernen Individuums an die empirische Allgegenwart kultureller Differenz binden. Dies geschieht explizit im Rahmen versuchter Beschreibungen der Globalisierung mit zwei unterschiedlichen Bezugspunkten. Erstens haben empirische Untersuchungen längst gezeigt, dass die selbstbezogenen Kognitionen und Emotionen von Angehörigen unterschiedlicher Kulturen jeweils verschieden ausfallen - wir also von kultu- 
rell variablen Selbstverhältnissen sprechen müssen, von denen nicht alle auf die Stärke des Individuums setzen. Als paradigmatisch galt hier lange Zeit die von den Kulturpsychologen Hazel Markus und Shinobu Kitayama (1991) in Analogie zur Scheidung «individualistischer» von «kollektivistischen» Kulturen eingeführte Differenzierung zwischen einem «independenten» und einem «interdependenten» Selbst. Nach der Logik dieses dichotomen Modells besteht der normative kulturelle Anspruch an das Selbst in «kollektivistisch» orientierten Kulturen eher darin, Interdependenz aufrecht zu erhalten als darin, sich getrennt und unabhängig vom sozialen Kontext eine eigenständige «Identität» zu geben. Individuelle, auf Individuation und auf die Verwirklichung der «eigentlichen» Bedürfnisse der Person angelegte Aspekte des Lebensentwurfs dürften damit kaum mehr als unzweifelhaftes Kriterium für «gelingende» Identität gelten (vgl. Kakar, 2006). Zweitens drängt auch die Realität der transkulturellen Gesellschaften auf eine weniger selbstidentische, stärker dezentrierte Vorstellung des personalen Selbst. Dabei geht es nicht allein um die als defizitär konstruierte Biografie jener Menschen «mit Migrationshintergrund», sondern vielmehr müssen sich heute alle Gesellschaftsmitglieder aufeinander zu bewegen, um eine sozial und kulturell differenzierte und in diesem Sinne globalisierte Gesellschaft zu ermöglichen. Allerdings sind nicht alle Angehörigen moderner, «westlicher» Gesellschaften vom kulturellen Wandel in gleichem Masse betroffen. Menschen, die über lang anhaltende Migrationserfahrungen verfügen, so etwa Said (1999), gelangen irgendwann in ein Stadium unentwegten Übersetzens und «Übersetzt-Werdens» und damit verbiete sich jedwede Festlegung auf eine Identität (oder auch auf mehrere festschreibbare Identitäten). Als akzeptable Alternative gilt ein flüchtiger Zustand des «dazwischen», ein diasporisches, hybridisiertes Selbst, welches immer im Übergang, im Zwischenraum ist (Appadurai, 1996). Die herrschaftskritische Perspektive der Subjektkritik findet also auch Eingang in die transkulturellen Diskurse zum Selbst, wenn sie sich gegen Identität verwahren, wo diese - vielleicht unter dem Deckmantel klinisch-psychotherapeutischer Kriterien - übergestülpt oder gar gewaltsam verfügt wird.

Die hier skizzierten Argumente bringen ökonomische, politische und gesellschaftliche Entwicklungen auf nachvollziehbare Weise mit psychischen Zuständen in Zusammenhang. Als sozialwissenschaftlich informierte Zeitdiagnosen stellen sie auch in ihren radikalen Schlussfolgerungen eine ernste Herausforderung dar, insofern sie zentrale Aspekte des kulturellen und sozialen Wandels pointiert ins Blickfeld rücken und problematisieren. Aber was folgt daraus für die akademische und die angewandte Psychologie, insbesondere die Psychoanalyse, die als 
wissenschaftliche Theorien und Praxen den Menschen in seiner Entwicklung und in der Gesellschaft zu ihrem Gegenstand haben? Wie kann die institutionalisierte (psychoanalytische) Psychotherapie diese Problematisierung des auf die Einzelperson zurechenbaren Selbst ernst nehmen - ohne sich als ethnozentrisches «Subjektivierungsinstrument» verstehen zu müssen, das Menschen dazu verhilft, die in neokapitalistischen Gesellschaften eingeforderte Flexibilität in ihrem Selbstund Weltverhältnis zu erlangen? Was haben die psychologischen Disziplinen dieser Kritik entgegen gehalten?

\section{Die Alternative der sozialkonstruktionistischen Psychologie}

Die akademische Psychologie widmet derartigen Zeitdiagnosen nicht allzu viel ernsthafte Auseinandersetzung. Nach wie vor dominiert vor allem in der kognitivistisch informierten Sozialpsychologie das Modell des individuell informationsverarbeitenden, rational handelnden Akteurs. Allenfalls an den Rändern der Disziplin versuchen sich sozialkonstruktivistische oder kulturpsychologische Ansätze an der Kritik dieses Menschenbilds, das sie auch längst auf Schlagworte gebracht haben: Kognitivismus, Rationalismus, Individuozentrismus sind wohl die gängigsten (vgl. Bruner, 1990; Gergen, 1999).

Der nordamerikanische Sozialpsychologe Ken Gergen hat dieser Kritik einen Gegenentwurf folgen lassen, den er als relational selfbezeichnet. Seine Vorstellung des in Beziehungen aufgehenden Selbst vermeidet den individuozentrischen «Engpass» und stellt einen nicht «subjektivierten», sondern in und durch sozialen Diskursen und Praktiken «konstituierten» Akteur in Aussicht. Mit Hinweis auf die Lebenswelt in globalisierten, spätmodernen Gesellschaften zeichnet er ein Bild des in Beziehungen eingebetteten Selbst. «Sich selbst» nurmehr als «Knotenpunkt in einer Verkettung von Beziehungen» zu verstehen, argumentiert er, könne das Dasein bereichern, unsere Identität werde damit «vielschichtiger», voll von inneren Dialogen, und die Kluft zwischen Selbst und Anderen werde gleichsam «in uns» aufgehoben: "Self and other are locked together in the generation of meaning" (1999, S. 131). In ähnlich idealisierender Perspektive beschreiben die niederländischen Psychologen Hubert Hermans und Harry Kempen (1993) oder der Psychoanalytiker Philip Bromberg (1996) die innere Struktur des wie sie sagen «dialogischen Selbst» und setzen unter Bezug auf die narrative Struktur moderner Identität darauf, dass analog zu den kulturell differenten Kontexten, in denen sich der oder die Einzelne heute bewege, eben unterschiedliche Erzählerpositionen «im» Selbst nebeneinander existierten. Auch der Münchener Sozialpsychologe Heiner Keupp beklagt zwar die «Entbettung» des Individuums in der reflexiven Moderne, instituiert aber 
zugleich einen euphemisierenden Begriff der «Patchwork-Identität», der sich diesen Bedingungen «anpasst» (Keupp, Ahbe \& Gmür, 2002).

Diese Alternativvorschläge aus psychologischer Feder nehmen die Herausforderung an, aus dem Individuozentrismus der Psychologie auszuscheren und eine zeitgemässe Konzeptualisierung des Selbst vorzuschlagen. Allein sie geraten dort zur Überzeichnung, wo das Kind mit dem Bade ausgeschüttet wird: Mit dem Ziel, die Konzeption eines Subjekts zu dekonstruieren, das zu rationalistisch und individualistisch gedacht erscheint, verabschiedet man kurzerhand die Idee des kohärenten Selbst schlechthin. Empirisch sieht das Bild anders aus. Zahlreiche Studien machen eher sichtbar, dass es für Heranwachsende und Erwachsene wichtig bleibt - unter postmodernen Bedingungen vielleicht wichtiger denn je ist -, die Heterogenität möglicher Lebensorientierungen zu reflektieren und entsprechende Übersetzungsleistungen zu erbringen, die ihnen diesbezüglich eine Wahl ermöglichen (Dornes, 2010; Bohleber, 2012). Identitätstheoretisch ist diese Radikalität nicht notwendig: Autonomie und Kohärenz sind nicht gebunden an ein vereinzeltes Ich, sondern können von Heteronomie durchkreuzt und in diesem Sinne als Prädikate eines in Beziehungen eingebundenen Selbst beschrieben werden. Die theoretischen Folgen der Absage an ein individuelles und einheitliches Selbst für die sozialwissenschaftliche Theoriebildung sind weitreichend: Geht man von einem vollständig sozial konstruierten Selbst aus, ist es gar nicht mehr möglich oder notwendig, nach denjenigen psychischen Prozessen zu fragen, die etwa die «Internalisierung» der vielfältigen Beziehungserfahrungen und mithin das Bilden von Selbst- und Objektrepräsentanzen erklären würden - gehören diese Prozesse doch zu jenem Individuum, das als Untersuchungsgegenstand verschwindet! Ein solches Ausklammern der individuell-selbstbezogenen Funktionen erscheint zunächst stringent. Auf den zweiten Blick werden aber ohne einen Begriff des individuellen Selbst auch sozialpsychologische Konzepte wie Beziehung oder gar Gesellschaft problematisch - bis es letztlich nur noch das Spiel der Zeichen, nur «Diskurs», «Praxis» oder «System» gibt. Diese und ähnliche theorieimmanente Kritikpunkte sind vielerorts vorgebracht worden (Honneth, 2000; Straub \& Zielke, 2005; Bohleber, 2006).

Resümieren wir an dieser Stelle kurz: Die zitierten Vorschläge aus den Reihen der Psychologie affirmieren - in dekonstruktivem Gestus - den «flexiblen Menschen» und die scheinbar notwendige Negation des Selbst. Sie tun dies in rhetorischer, z. T. euphemisierender Form, indem sie den Verlust an Identität in eine ästhetisch-spielerische Variante von «Freiheit» verklären. Mit den Risiken, die da lauten: Naivität (der Theorie), Beliebigkeit (der Lebensvollzüge), Verlust 
(des kritischen Subjekts), versöhnt man sich, da nur so dem Scheitern der grossen Erzählungen vom Subjekt der Aufklärung Rechnung getragen wird. Wie alle postmodernistisch argumentierenden Diskurse lebten auch die skizzierten Positionen davon, sich nur gegen eine Extremposition zu begründen und im Weiteren behaupten zu können. Für die genannten akademisch-psychologische Arbeiten mag eine solche Extremposition durch die hartnäckige Trägheit der Mainstream-Psychologie vorliegen, die - nicht zuletzt aufgrund ihrer naturwissenschaftlichen Ausrichtung am neopositivistischen Programm nomothetischer Forschung - seit den 1950er Jahren alternative Diskurse und Forschungen an den Rand drängt und sich tatsächlich immer wieder gewissen «Engpässen» der Moderne unterordnet. Für die Psychoanalyse kann das so aber nicht gelten.

\section{Die Zwischenposition der Psychoanalyse}

Aus einem allgemeinen Blickwinkel zeichnet die Psychoanalyse von jeher das Bild eines Menschen, der nicht Herr im eigenen Hause ist, dem eine gewisse innere Zerrissenheit auch jenseits pathologischer Selbstverhältnisse zugesprochen wird. Liegt sie damit nicht ohnehin und eigentlich schon immer quer zu den gängigen Subjektmodellen, gegen die sich die Kritik der Moderne richtete? Für diese Vermutung spricht das Aufgreifen der Psychoanalyse durch die Kritische Theorie eines Adorno, Horkheimer oder Marcuse, aber auch durch jüngere, den postmodernen Thesen der Auflösung des Subjekts näher stehende Autoren und Autorinnen (z. B. Butler, 1989; Derrida, 1996). Die Bedeutung, die den unbewussten Elementen des Wissens und ebenso den unbewussten Motiven des Handelns zukommt, hat die Psychoanalyse seit ihren Anfängen als einen Gegenentwurf zur typisch «modernen» Fixierung auf Rationalität und Autonomie, auf Integrität und Kohärenz prädestiniert. Bereits Freud habe die Einheit des Psychischen radikal in Frage gestellt und die Vorstellung vom Subjekt als Souverän seines Handelns desavouiert, schreibt der Psychoanalytiker Klaus-Jürgen Bruder. Und er folgert prägnant: «Für eine Kritik der Moderne müssen wir also von der Psychoanalyse ausgehen» (2007, Abschn. 1). Der Abstand des psychoanalytischen zum klassisch «modernen» Subjekt ist also leicht auszumachen! Auf den zweiten Blick aber scheinen Widersprüchlichkeiten auf: Denn zugleich ist eben diese Psychoanalyse mit vielfältigen Spezifika der Moderne eng verknüpft. Sie ist ja selbst entstanden als Antwort auf eine veränderte Lebensführung, die sich in der Neuzeit durchzusetzen begann. Als eine unter anderen institutionalisierten Praktiken gibt sie Zeugnis von und trägt selbst bei zu jenen kulturellen Entwicklungen, die später als Verzeitlichung, Individualisierung und Autonomisierung benannt werden konnten. 
Entwicklungen, welche die Subjektivität des und der Einzelnen enorm aufwerteten und damit erst zu einem prekären Thema der eigenen Lebensführung machten. Die soziologische Biografieforschung hat schon vor Jahrzehnten das psychoanalytische Erstgespräch als modernen «Biografiegenerator» (Hahn, 1987), mithin als eine der institutionalisierten Praktiken gekennzeichnet, die konstitutiv dafür waren, dass sich eine typisch neuzeitliche, «diachrone» Identität überhaupt ausbilden konnte. «Ob das Ich über Formen des Gedächtnisses verfügt, die symbolisch seine gesamte Vita thematisieren, das hängt vom Vorhandensein sozialer Institutionen ab, die eine solche Rückbesinnung auf das eigene Dasein gestatten» (Hahn, 1987, S. 12). Die klassische Psychoanalyse, der es um die intrapsychische Struktur der Person geht, ist eine solche Institution. Durchaus im Foucaultschen Sinne ist sie Teil desjenigen Dispositivs, also desjenigen Bündels an Diskursen und Praktiken, die dazu führten und zugleich selbst darauf bauten, dass wir heute dem und der Einzelnen die Aspiration auf einen (mehr oder weniger selbstbestimmten und selbst verantworteten) Entwurf des eigenen Lebens zuschreiben. Die Psychoanalyse ist also auch eine kulturelle, westliche, moderne Praxis (vgl. Zielke \& Straub, 2007).

Als westlich-moderne Praxis hat auch die Psychoanalyse in ihrer Metapsychologie ein individuozentrisches Fundament. Intersubjektive Perspektiven hatten lange Zeit keinen guten Stand, und dies obwohl es, wie allgemein bekannt, schon seit den 1920er Jahren Theoretiker gab, etwa Ferenczi, die eine intersubjektive, beziehungsorientierte Richtung vertraten. Für Freud war die Frage nach der sozialen Identitätsbildung nicht im Vordergrund - er befasste sich mit einem weitgehend intrapsychischen Reifungsprozess, in dem Beziehungen zu anderen Personen nur durch ihre enge Verbindung mit Trieben wichtig waren. Eine ernsthafte Auseinandersetzung mit dem Begriff der Identität vermachte bekanntlich Erik Erikson der Psychoanalyse in den 1940er Jahren: Unter Rückgriff auf die in den USA bereits einschlägigen philosophischen Diskurse und Forschungen zum Selbst verstand er den Begriff bereits sozialpsychologisch, also im Sinne der pragmatistisch und intersubjektiv verfassten Identitätstheorie bei Mead (1935). Jedoch erst die Objektbeziehungstheorie und insbesondere die Vertreter der «middlegroup» sahen den Stellenwert der frühkindlichen Beziehungen und der affektiven Bindung an virtuelle und konkrete Andere für das Gelingen des kindlichen Reifungsprozesses als so zentral an, dass sich auch die Vorstellung vom Selbst ent-individualisierte. Als Vorläufer dieser Entwicklung in der Psychoanalyse gelten neben Ferenczi Loewald, Kohut, Balint und Winnicott. Seit den 70er Jahren haben die empirische Säuglingsforschung auf der einen und philosophische Ansätze auf der anderen Seite wichtige weitere Anstösse «von aussen» gegeben. Heute wird von 
der relationalen Analyse eine "primäre Intersubjektivität» postuliert, in der sich die einzelnen intentional Handelnden nicht ohne Weiteres voneinander abgrenzen lassen (müssen) (Mitchell, 1993; Ogden, 2006a; Orange, Atwood \& Stolorow, 1997). Erst damit hat man sich von der Vorstellung des individuellen Geistes als klar abgrenzbarer Entität wirklich entfernt - und die Rede vom «intersubjektiven Paradigma» wird von einem grossen Lager der ansonsten sehr diversen internationalen Psychoanalyse angenommen.

Hier drängt sich die Frage auf - wenngleich dies den Rahmen der vorliegenden Abhandlung sprengen würde -, wieso die Psychoanalyse die lebendige sich entwickelnde philosophische Intersubjektivitätsdebatte (von der Phänomenologie Hegels und Husserls über Meads Symbolischen Interaktionismus zu Habermas' Sozialphilosophie) lange nicht zur Kenntnis nahm. Sollte auch hier neben der kulturellen Affinität zum Individualismus eine - ebenfalls zu den grossen Erzählungen der Moderne gehörende-positivistische Erkenntnishaltung für einen blinden Fleck in der Psychoanalyse gesorgt haben? Dafür spräche, dass die (philosophische) Intersubjektivitätstheorie ja bis heute nur im Verbund mit den Erkenntnissen der Säuglingsforschung akzeptiert wird, die deren Aussagen nun experimentell nachspielt und «überprüft». Immer wieder macht es stutzig, wie der Säuglingsforschung selbstverständlich der Vorrang beim Vorbereiten des neuen Paradigmas eingeräumt wird, so etwa bei Werner Bohleber, wenn es über die Bedeutung früher Intersubjektivität und vorsprachlich repräsentierter intersubjektiver Erfahrungen heisst: «Einer solchen Entwicklung hat anscheinend weniger die Philosophie mit ihrer Phänomenologie von Selbst und Anderem als vielmehr die Säuglingsforschung den Weg geebnet» (2006, S. 222).

Zum Gewinn der intersubjektiven Perspektive innerhalb der Psychoanalyse existiert vielfältige Literatur (zum Überblick vgl. Altmeyer \& Kächele, 2006). Ich will an dieser Stelle - ohne Anspruch auf Systematik oder Vollständigkeit - versuchen zu zeigen, in welcher Hinsicht und wodurch die psychoanalytisch informierte Intersubjektivitätstheorie von der sozialwissenschaftlichen lernen, aber auch und vor allem, worin sie etwas Zusätzliches beitragen kann.

\section{Herausforderungen für eine globalisierte Psychoanalyse}

Psychoanalyse als individualisierender Biografiegenerator: Es liegt auf der Hand: Nicht trotz, sondern wegen verstärkter Differenzanforderungen hat die Psychoanalyse die Funktion eines geheimen Ortes, wie etwa die Beichte (Hahn, 1987). Eines Ortes, an dem das ansonsten dezentrierte Individuum als Ganzes thematisch werden darf - im Gegensatz zur öffentlichen Realität, in der es sich 
jeweils als der eine oder die andere konstruieren muss. In diesem Sinne wirkt sie individualisierend, weil sie den haltgebenden Rahmen bietet, die Pluralität und Heterogenität und oft auch die Widersprüchlichkeit möglicher Handlungs- und Erlebensoptionen in der Lebenswelt des und der Einzelnen zu reflektieren, und weil in diesem Rahmen die nötigen Übersetzungsleistungen zwischen solchen unterschiedlichen Kontexten vorbereitet werden, die es - in den meisten Fällen ermöglichen, sich selbst in diesen unterschiedlichen Entwürfen wiederzuerkennen.

Es wird immer wieder deutlich, dass gerade für die Artikulation und die rekonstruktive Bearbeitung von Differenz zumindest die Aspiration, der Wunsch nach einer kohärenten personalen Identität, nach einem eigenen - vielleicht hybriden - Selbstentwurf unabdingbar ist. Möglicherweise ist es das, was der Psychoanalytiker Philip Bromberg benannt hat als die «Fähigkeit, in den Räumen zwischen unterschiedlichen Realitäten zu stehen, ohne eine davon zu verlieren» - die Fähigkeit, sich als ein Selbst zu fühlen, während man doch eine Vielheit ist (1996, S. 4; Übers. B.Z.). Bromberg geht es dabei um die (angestrebte) Fähigkeit beider, sich auch auf jene subjektiv wahrgenommene und erlebte Identität einzulassen, die sich nicht ohne Weiteres einfügt in die bewusste Selbstrepräsentanz des Analysanden oder Theorie des kohärenten Selbst, der die Psychoanalytikerin anhängt. Die vielleicht sogar als Nicht-Identisches auftritt (wie etwa besonders prägnant im Falle der transgenerationellen Übertragung von Traumatisierungen, die sich im Selbst als Fremdes zeigen und auch in der Behandlungssituation, vgl. z. B. Moré, 2013.) Diese Form der kohärenten personalen Identität kann auch heute noch Ziel von Psychoanalysen sein - und ist es wohl auch. Etwas zugespitzt könnte man sagen, in vielen Fällen beginnt die psychoanalytisch-therapeutische Arbeit am Selbst genau dort, wo die rein sozialwissenschaftlich konzipierten Modelle des dezentrierten Selbst oft enden: nämlich mit der Auflösung jeglichen Einheitsgefühls. Genau damit beginnt nicht nur die theoretisch interessante Frage nach einer spätmodernen Identität, sondern auch in vielen Fällen der konkrete Auftrag analytischer Psychotherapien (Zielke \& Straub, 2007).

Die sozialphilosophische Intersubjektivitätsdebatte hat Identität als Prozess und Ergebnis kommunikativer Selbstvergewisserung beschrieben (Habermas, 1992). Damit ist Identität nicht nur sozial konstituiert, sondern auch eine höchst fragile Angelegenheit: Identität als kommunikative Selbstvergewisserung ist die Einheit ihrer Differenzen und damit niemals gegeben. Wie Renn und Straub (2005) formulierten: Spätmoderne Identität ist eine Aspiration und damit kein jemals erreichbarer Zustand, sie ist transitorisch, sie bildet und bewährt sich im Übergang und in der Veränderung ihrer selbst. 
Die entwicklungspsychologische Perspektive: Die psychoanalytische Intersubjektivitätsdebatte hat die Entstehung des Selbst entwicklungspsychologisch in frühere Lebensphasen verlegt. Sie befasst sich seit Jahrzehnten mit den Bedingungen seiner Entstehung zwischen primärer Bezugsperson und Säugling. Was ich bin und was nicht, entsteht in der Auseinandersetzung mit primären und weiteren Anderen, und zwar von der ersten Minute der ontogenetischen Entwicklung an. Damit hat die psychoanalytische Theoriebildung zum Selbst eine Frage selbstverständlich immer mitgedacht, die in den Vorschlägen aus Soziologie oder auch im Sozialkonstruktivismus Gergens mit dem Argument einer verzerrenden Fixierung aufs Individuum ausgeklammert wurde: die Frage der «Internalisation», die Frage danach, wie der Austausch mit dem virtuellen und den konkreten Anderen sich als psychische Repräsentanz im Selbst zeigt.

Vielfältige Konzepte über die Form der internen Repräsentation früher Beziehungserfahrungen sind auf diesem Weg elaboriert worden, die das Denken über eine soziale oder relational verfasste Identität bereichern können. Die Untersuchungen dazu, wie die frühen Versuche des Kindes verlaufen, selbstständig gegen die Mutter zu werden, führt schlüssig zur Grundannahme, dass der Prozess der «Internalisierung» der Beziehungen mit Anderen nicht nur sehr komplex ist, sondern auch jeweils unterschiedlich verläuft: nämlich auf der Basis eines früh gebildeten, vorsprachlichen sozialen Erfahrungsmodus, der als ein unbewusstes Miteinander den Modus der weiteren Identitätsbildung prägt. Die frühkindliche Entwicklung ist in eine primäre Umweltbeziehung eingebettet, die sich als «Schatten des Objekts» (Freud, 1917) in der Struktur des sich entwickelnden Selbst festsetzt, das Kind hat mit dem «unthought known» (Bollas, 1997) ebenso wie mit dem «Nicht-Wissen» (Winnicott, 1965) eine im Körpergedächtnis repräsentierte Gewissheit über eine selbstverständliche «haltende Umwelt». Auch in Ogdens (1992) «autistisch-berührender» Position geht es um diese implizite, frühe Gewissheit, die sich an der sensorischen Erfahrung mit dem/den primären Objekt/ en festmacht. Die damit eingenommene Perspektive auf die Entstehung des Selbst aus Beziehungen bildet einen Mehrwert, der den genannten sozialwissenschaftlichen Modellen fehlt.

Überdies kann die Psychoanalyse durch die Fokussierung dieser frühen, leiblich repräsentierten Basis der «Internalisierung» auf die emotionale Qualität hinweisen, mit der diese implizite Gewissheit im individuellen Fall erworben wurde. Diese ist die massgebliche Matrix dafür, wie und unter welchen Vorzeichen im weiteren Leben die Beziehungen mit konkreten Anderen internalisiert werden. Auch Stephen Mitchell fügt seiner Auffassung, dass das Selbst aufgrund unter- 
schiedlicher Beziehungserfahrungen intern «vielfältig» sei (1993, S. 104) nachdenklich hinzu: «Die Beziehungen zwischen diesen unterschiedlichen Versionen oder Organisationen des Selbst sind komplex und sie sind in hohem Masse davon abhängig, wie stark die Konflikte und wie verlässlich die Loyalitäten in den realen, äusseren Beziehungen waren, aus denen sie sich entwickelt haben» (1993, S.104, Übers. B.Z.). Objektbeziehungstheoretisch geht es hier darum, welche inneren Objekte mit wie un/verdaulichen Beziehungsangeboten «in » einem Selbst präsent sind und agieren - dies verleiht dem dezentrierten Selbst einen bestimmten «Modus» zur (ebenfalls dezentrierten) Welt hin.

Worin dieser affektiv konnotierte, implizite Modus «hinter» den unterschiedlichen thematischen Varianten eines Selbstentwurfs besteht, zeigt sich und wird herausgearbeitet im psychoanalytischen Prozess, er wird für die Analytikerin oft körperlich spürbar, lange bevor sie in der Lage ist, die damit reinszenierte Stimmung verbal einzufangen und mit dem Patienten zusammen auf sein gelebtes Leben zu beziehen. Das Zusammenspiel von Reflexion und Praxis des Selbst stellt eine weitere Besonderheit der psychoanalytischen gegenüber den sozialwissenschaftlichen Konzeptualisierungen dar.

Das produktive Pendeln zwischen Theorie des Selbst und Theorie der Behandlung: Sprechen psychoanalytische Theoretiker vom intersubjektiven Paradigma, haben sie in aller Regel zweierlei im Sinn - die mehr und mehr intersubjektiv verstandene Genese des Selbst aus der Interaktion mit anderen und die immer stärker intersubjektiv und relational verstandene Behandlungssituation. Damit ist die «reine» Theoriebildung des Selbst über den Bezug zur psychoanalytischen Praxis immanent an eine konkrete soziale Situation gebunden, in der es um dieses Selbst geht: Beide Ebenen werden aufeinander bezogen und im Rahmen der Rekonstruktion der Identität des Analysanden, die sich im Verlauf des analytischen Prozesses weiterentwickelt, in einer Art Pendelbewegung miteinander verbunden. Viele derjenigen Prozesse, die für die Bildung des Selbst postuliert werden, werden auch für die Technik der Behandlung als relevant erachtet und theoretisch elaboriert. Es existiert mittlerweile eine Vielfalt an Konzepten, die aus dem je spezifischen Verständnis der Mutter-Kind-Interaktion für die Behandlungssituation entwickelt wurden bzw. in ihr Anwendung finden, so etwa «Containment» oder für neuere Theorien des Selbst und des psychoanalytischen Prozesses eben: «Dialogizität». Damit wird die Kluft zwischen Empirie und Metapsychologie verringert - allerdings ohne den Weg der scheinbaren Objektivität nomologischer Methoden zu beschreiten. 
In den entsprechenden Untersuchungen der Übertragungs-Gegenübertragungsszenarien rücken aus relationaler Sicht diejenigen Aspekte in den Vordergrund, bei denen die handelnden Einzelnen in den Hintergrund rücken. Der interaktionelle Prozess selbst wird vorrangig, als bedeutungskonstitutives Feld, das in der Dichotomie von Ego und Alter nicht aufgeht. Es geht um enactments, projektive Identifizierungen, Rollenübernahmen - Konzepte, in denen die vormals den Akteuren «subjektiv» zugerechneten Aspekte von Übertragung und Gegenübertragung (etwa deren Emotionen, Kognitionen, sensorische Perzeptionen, Handlungen usf.) als Bestandteile eines mit einer eigenen Dynamik ablaufenden Prozesses verstanden werden. Das «inter» rückt in den Fokus, ihm werden - sofern man überhaupt noch von Intersubjektivität spricht - in manchen Beschreibungen nachgerade jene Intentionalität und jene Autonomie zugesprochen, die dem Einzelsubjekt abgesprochen werden! Der Übergangsraum, den Winnicott als entlastendes «Zwischen» für Kleinkind und Mutter konzipiert hatte, wird zum «analytischen Dritten» (Ogden, 2006b) in der Behandlungssituation die damit den Beschreibungen einer «sozialen Praxis» im Bourdieuschen Sinne genügt. Psychologisierender ausgedrückt: «Es geht um Bezogenheitsprozesse per se» (Bohleber, 2006, S. 218). Das klingt wirklich nach einem paradigmatischen Perspektivenwechsel. Aber wo bleibt bei dieser Konzeption des intersubjektiven Prozesses das Selbst? Auch in der intersubjektiven Psychoanalyse geht es jetzt um die Frage, wie diese Konzeption der sozialen Praxis die Partizipienten und ihre Intentionalität und eben auch: ihr je individuelles Selbst überhaupt noch «denken» kann - ohne durch die Hintertür neue Subjektivismen hereinzubitten (indem, etwa wie in der kleinianischen Objektbeziehungstheorie, alles, was überhaupt in der Analyse passiert, in den Bereich intrapsychischer «unbewusster Fantasien» verlegt wird). Thomas Ogdens Selbstkorrektur, mit der er dem «analytischen Dritten» den zunächst eingeräumten «autonomen» Status wieder entzieht und nun von der «Erfahrung des unbewussten Zusammenspiels von (getrennter) Subjektivität und (geteilter) Intersubjektivität» spricht (2006b, S. 37), zeigt, dass die Psychoanalyse nun wirklich im intersubjektiven Paradigma angekommen ist - und damit auch bei dessen inhärenten begrifflichen Problemen! Dies gilt auch für die Frage, wie die ko-konstruierten Bedeutungen in der Welt verankert sind.

Psychoanalytisches Wissen zwischen Konstruktion und Realität: Mitchell (1993; 2005) hat betont, dass der Paradigmenwechsel zur Intersubjektivität nicht nur eine veränderte Auffassung des Selbst, eine Revolution der Behandlungstechnik, sondern auch einen explizit epistemologischen Aspekt beinhalte: die Infragestellung des sicheren Status des psychoanalytischen Wissens. So argumentiert auch 
Hoffmann, der vom Wechsel der Epistemologie von einem positivistischen hin zu einem konstruktivistischen Modell der psychoanalytischen Situation spricht (1998); Schafer (1985) beschreibt den psychoanalytischen Prozess als eine gemeinsame (narrative) Konstruktion. Es wird immer häufiger betont, dass das, was hier gemeinsam «erkannt» wird, weder Analysand noch Analytikerin allein zugänglich ist, die gewonnenen Einsichten sind per definitionem relational - und damit fragil, implizit, beweglich. "In this transitional space", schreibt Bromberg, "reality is a shared mental state - a channel of implicit communication" (2012, S. 97).

Diese radikal sozialkonstruktivistische Auffassung ist zunächst nachvollziehbar: Bezogen auf das Selbst der Person ist die psychoanalytische Therapie erst einmal auch nur ein weiterer relationaler Kontext, in dem sich die kontingenten Elemente eines Selbstentwurfs bilden. Dies geschieht in mehr oder weniger bewussten kommunikativen Prozessen, in denen es um geteiltes, unbewusstes und implizites «Wissen» geht (paradigmatisch sind die zwischen den Interaktanden «ablaufenden» szenischen Momente wie enactments oder now-moments). Das dabei entstehende fallspezifische Wissen bleibt zunächst im Besitz des jeweiligen analytischen Paares, das dieses Wissen aber auch nicht «hat», sondern es immer wieder aufs Neue bilden muss. Die Festschreibung dieser impliziten Wissensbestände qua Verbalisierung ist nur unter Verlusten möglich bzw. muss selbst wieder als problematische Übersetzung von implizit nach explizit gelten, für die es allenfalls ein selbst äusserst kontingentes, vielleicht hypothetisches tertium comparationis geben kann (Renn, 2006). Das psychoanalytische Wissen ist damit schon in seinem Entstehungskontext unsicher und flüchtig, wenn es verbalisiert und «festgeschrieben» wird, ist erst recht von Verzerrungen und Ausblendungen auszugehen. Diese sind mitzudenken - universalisierende Objektivitäts- und Wahrheitsansprüche stehen per definitionem im Verdacht verkürzender Reifizierung. Bis hierhin ist auch der (postmodern orientierten) Wissenskritik zuzustimmen.

Aber bleibt die Realität damit tatsächlich «draussen»? Wo bliebe dann der Raum für das erfahrene Leid, für die Angst vor der Auflösung, für die maligne wirksame Beziehungsrepräsentanz, für den quälenden Wiederholungszwang? Mit dieser Frage klopfen wir an die Tür zur philosophischen Konstruktivismuskritik, die wir aus Platzgründen geschlossen lassen müssen. Gleichwohl lässt sich auch in der hier gebotenen Kürze sagen: Auch der noch so flüchtige, implizit und unbewusst sich (selbst-)steuernde Dialog zweier Partizipienten (bei dem im Übrigen auch noch viele virtuelle «Andere» mit im Raum sind) «funktioniert» nur über eine in der Realität verankerte soziale «Praxis» oder Sprachgemeinschaft (Cavell, 2006). Eine psychoanalytische Behandlung ist keineswegs ein Dialog im luftleeren Raum, das 
wollen auch die Vertreter der narrativen, relationalen Konstruktion nicht so sehen (Schafer, 1999). Vielmehr liefern der Bezug zur Theorie der Behandlung über die leibliche Präsenz des Analytikers und der Patientin (die dem selben Raum «einwohnen») und die auch sinnlich wahrgenommene Gegenübertragung der psychoanalytischen Theoriebildung starke Argumente gegen den Vorwurf der Beliebigkeit. Damit kann sie im Einzelfall auf die Störbarkeit der Objektbeziehungen hinweisen und deutlich machen, dass auch das aus Intersubjektivität emergierende Selbst an Existenzbedingungen gebunden ist und dass es - wenngleich auch die Kriterien hierfür kulturell variabel sind - scheitern kann.

\section{Resümee}

Die heutige Psychoanalyse verfolgt in weiten Teilen die Genese des Intrapsychischen aus dem Intersubjektiven. Die Frage nach dem Unbewussten wird zur Frage nach dem imaginären und virtuellen Anderen, der immer mitgedacht wird, wenn wir unser Selbst (neu) entwerfen. Um dies zu ermöglichen, muss die Psychoanalyse sich selbst als relationalen Kontext verstehen, sie muss diskursiver Raum und geteilte Praxis sein. Dafür stehen die neueren Arbeiten zur relationalen Analyse ein.

Das Selbst wird zu einem unabschliessbaren Projekt und auch zu einer sozialen Konstruktion - zunächst durchaus im «postmodernen» Sinne. Identität wird zum Übergang von einem Status der Person zum nächsten, zur Transformation ihrer Selbst zur transitorischen Identität (Renn \& Straub, 2005), die sich von Anfang an im sozialen Raum, also partizipatorisch bildet. Die intersubjektiven Ansätze der Psychoanalyse antworten mit dieser Konzeptualisierung von Identität auf die Anforderung globalisierter Lebenswelten an das Selbst, in der Vielfalt möglicher und voneinander getrennter Beziehungen realer, mediengestützter oder virtueller Art doch noch ein einheitliches Selbstgefühl aufrecht zu erhalten. Der von sozialen Anderen mit-konstituierte und durchkreuzte «Sinn» der eigenen Geschichte, die ein solches transitorisches und partizipatorisches Selbst bildet, ist bei Lichte besehen tatsächlich nicht recht viel mehr als ein kontrafaktisch unterstellter normativer Fluchtpunkt des gelebten Lebens: Aber er ist nach wie vor Gewähr dafür, dass es uns bei unseren vielfältigen und widersprüchlichen Handlungen und Unterlassungen um etwas geht.

Mit diesem Identitätskonzept darf sich auch eine zeitgemässe Psychoanalyse noch als Möglichkeit der kritischen Aneignung des eigenen Lebens verstehen - die von den Kritikern des kohärenten Selbst angeführten Einwände verbieten allein die Fixierung und klare Benennung des Zieles dieser Reise. Das meinen wohl auch Aron 
und Harris, wenn sie - hier mit Blick auf die Identität der Psychoanalyse - konstatieren: «Im Fluss zu bleiben, ohne zu zerfliessen, das ist die hohe Kunst» (2006).

\section{Literatur}

Aron, L. \& Harris, A. (2006). In Beziehungen denken - in Beziehungen handeln. In M. Altmeyer \& H. Thomä (Hrsg.), Die vernetzte Seele (S. 108-121). Stuttgart: Klett-Cotta.

Baudrilliard, J. (1976). L'Échange symbolique et la mort. Paris: éd. Gallimard, Paris. (Der symbolische Tausch und der Tod. München: Matthes \& Seitz).

Bauman, Z. (1996). From Pilgrim to Tourist. In S. Hall \& P. du Gay (Hrsg.), Questions of Cultural identity (S.17-25). London: Sage.

Beck, U. (1986). Risikogesellschaft. Auf dem Weg in eine andere Moderne. Frankfurt a. M.: Suhrkamp.

Bohleber, W. (2006). Der Andere in der psychoanalytischen Tradition. In M. Altmeyer \& H. Thomä (Hrsg.), Die vernetzte Seele (S. 203-226). Stuttgart: Klett-Cotta. Bohleber, W. (2012). Was Psychoanalyse heute leistet. Stuttgart: Klett-Cotta.

Bollas, C. (1997). Der Schatten des Objekts. Stuttgart: Klett-Cotta.

Bromberg, P. (1996). Standing in the Spaces. The Multiplicity of Self And The Psychoanalytic Relationship. Contemporary Psychoanalysis, 32, 509-535.

Bromberg, P. (2012). The Shadow of the Tsunami and the Growth of the Relational Mind. New York: Routledge.

Bruder, K.-J. (2007). La condition postmoderne - est-ce-qu'elle est passée? Eine Zeitdiagnose. Online: http://foucault.de/macht.htm.

Brunner, J. (1990). Acts of Meaning. Cambridge: Harvard University Press.

Dornes, M. (2010). Die Modernisierung der Seele. Psyche, 11(64), 995-1033.

Foucault, M. (1989). Die Sorge um Sich. Sexualität und Wahrheit. In Bd. III. Frankfurt a. M.: Suhrkamp.

Freud, S. (1917). Trauer und Melancholie. In GW Bd. X(S. 427-446). Frankfurt a. M.: Fischer.

Gergen, K. (1999). Invitation to Social Construction. London: Sage.

Giddens, A. (1990): The Consequences of Modernity. Stanford: University Press.

Habermas, J. (1992). Individuierung durch Vergesellschaftung. Zu G. H. Meads Theorie der Subjektivität. In Ders., Nachmetaphysisches Denken. Philosophische Aufsätze (S. 187-241). Frankfurt a. M.: Suhrkamp.

Hahn, A. (1987). Identität und Selbstthematisierung. In A. Hahn \&V. Kapp (Hrsg.), Selbstthematisierung und Selbstzeugnis: Bekenntnis und Geständnis (S. 9-24). Frankfurt a. M.: Suhrkamp. 
Hermans, H. \& Kempen, H. (1993). The Dialogical Self. Meaning as Movement. San Diego: Academic Press.

Hoffman, I. Z. (1998). Ritual and Spontaneity in the Psychoanalytic Process. A Dialectical-constructivist View. Hillsdale: The Analytic Press.

Honneth, A. (2000). Objektbeziehungstheorie und postmoderne Identität. Über das vermeintliche Veralten der Psychoanalyse. Psyche, 54(11), 1087-1109.

Kakar, S. (2006). Kultur und Psyche - Auswirkung der Globalisierung auf die Psychotherapie. In Strauss, B. \& Geyer, M. (Hrsg.), Psychotherapie in Zeiten der Globalisierung (S. 163-181). Göttingen: Vandenhoeck \& Ruprecht.

Keupp, H., Ahbe, T. \& Gmür, W. (2002). Identitätskonstruktionen. Das Patchwork der Identitäten in der Spätmoderne. Hamburg: Rowohlt.

Mitchell, S. (1993). Hope and Dread in Psychoanalysis. New York: Basic Books.

Mitchell, S. (2005). Psychoanalyse als Dialog. Göttingen: Psychosozial Verlag.

Moré, A. (2013). Überantwortete Vergangenheitsbewältigung. Zur Bedeutung transgenerationaler Gefühlsvererbung, Psychologie \& Gesellschaftskritik, 4/12, $1 / 13,35-60$.

Ogden, T. (2006a/1989). Frühe Formen des Erlebens. Göttingen: Psychosozial Verlag.

Ogden, T. (2006b). Das Analytische Dritte, das intersubjektive Subjekt der Analyse und das Konzept der projektiven Identifizierung. In M. Altmeyer \& H. Thomä (Hrsg.), Die vernetzte Seele (S. 35-63). Stuttgart: Klett-Cotta.

Renn, J. \& Straub, J. (Hrsg.) (2005). Transitorische Identität. Der Prozesscharakter des modernen Selbst. Frankfurt a. M.: Campus.

Renn, J. (2006). Übersetzungsverhältnisse. Perspektiven einer pragmatistischen Gesellschaftstheorie. Frankfurt a. M.: Velbrück.

Rose, N.(1989). Governing the Soul. The Shaping of the Private Self. New York: Free Association Press.

Said, E. (1999). Out of Place. A Memoir. New York: Random House.

Sennet, R. (1998). Der flexible Mensch. Berlin: BtV.

Straub, J. \& Zielke, B. (2005). Autonomie, narrative Identität und die postmoderne Kritik des Sozialen Konstruktionismus. In F. Jäger, \& J. Straub (Hrsg.), Was ist der Mensch - was Geschichte? (S. 165-210). Bielefeld: transcript.

Zielke, B. \& Straub, J. (2007). Kultur, Psychotherapie und das kulturelle Selbst als transitorische Identität. Eine Erwiderung auf postmoderne und sozialkonstruktionistische Varianten «narrativer Therapie». Handlung Kultur Interpretation, 16(2), 286-316. 